EGU2020-18391

https://doi.org/10.5194/egusphere-egu2020-18391

EGU General Assembly 2020

(c) Author(s) 2021. This work is distributed under

the Creative Commons Attribution 4.0 License.

\title{
How obstacle geometry and snow properties influence avalanche impact pressure
}

\author{
Michael L. Kyburz ${ }^{1,2}$, Betty Sovilla ${ }^{1}$, Johan Gaume ${ }^{3}$, and Christophe Ancey ${ }^{2}$ \\ ${ }^{1}$ WSL Institute for Snow and Avalanche Research SLF, Davos, Switzerland (michael.kyburz@slf.ch) \\ ${ }^{2}$ Environmental Hydraulics Laboratory, Swiss Federal Institute of Technology, Lausanne, Switzerland \\ ${ }^{3}$ Snow and Avalanche Simulation Laboratory SLAB, Swiss Federal Institute of Technology, Lausanne, Switzerland
}

In order to estimate avalanche loads on buildings and structures of various sizes and geometries, practitioners are interested in recommendations or experimental data for a wide variety of obstacle geometries and sizes. Full-scale avalanche measurements are performed across the world since the late 1970s to increase knowledge about avalanche flow behaviour, including impact on structures. These structures are usually equipped with sensors to measure impact pressure, avalanche velocity and/or snow density. Modifying the structure profile is hardly possible because of high construction costs. To date, it has thus been possible to test and calibrate empirical relationships used in engineering only on a limited number of structures for which experimental data exist. We therefore aim to calibrate the drag coefficient and amplification factor for a broader range of obstacle shapes and sizes. In this context the drag coefficient generalizes the drag coefficient used in Newtonian fluid mechanics when computing the flow past an obstacle. The amplification factor reflects the snow load's deviation from a hydrostatic-like pressure. To estimate these two parameters, we simulate how an avalanche interacts with differently sized and shaped obstacles using the Discrete Element Method (DEM). First, we test the DEM model's capacity to reproduce full-scale pressure measurements performed on two different obstacles at the Vallée de la Sionne test site by comparing simulated and measured impact pressures. Second, we run new simulations involving other geometries and dimensions, for which no experimental data exist. Our results show that the pressure distribution depends not only on the obstacle geometry, but also on avalanche flow regime and snow properties. We eventually examine the pressure distribution for different generic geometries and avalanche scenarios. This analysis should ultimately help to improve extant engineering guidelines. 